\title{
Rectal Cancer in Common Variable Immunodeficiency: A Case Report
}

\author{
Vivian Regina Guzela ${ }^{1}$, Carlos Walter Sobrado Júnior ${ }^{1}$, Dewton de Moraes Vasconcelos ${ }^{2}$, Lucas Faraco Sobrado ${ }^{2}$, \\ Sérgio Carlos Nahas ${ }^{1}$ and Ivan Cecconello ${ }^{1}$
}

${ }^{1}$ Hospital das Clínicas da Faculdade de Medicina, Universidade de São Paulo, Departamento de Gastroenterologia, Brazil

${ }^{2}$ Faculdade de Medicina - Universidade de São Paulo, Instituto de Medicina Tropical de São Paulo, Brazil

Submission: March 03, 2020; Published: April 03, 2020

*Corresponding author: Vivian Regina Guzela, Hospital das Clínicas da Faculdade de Medicina, Universidade de São Paulo, Departamento de Gastroenterologia, Av. Dr. Enéas de Carvalho Aguiar, 255. Cerqueira César - São Paulo, SP 05403-000, Brazil

\begin{abstract}
Introduction: Common variable immunodeficiency (CVID) is a primary immunodeficiency related predominantly to immunoglobulin (Ig) $\mathrm{G}$ and IgA deficiency with or without changes in IgM levels. Other immunological pathways may also be altered, which makes this pathology extremely heterogeneous. The main manifestations include predisposition to severe infections, development of autoimmune diseases and greater susceptibility to the development of neoplasms, especially lymphomas and gastric cancer. There are reports of colorectal and anal cancer in this population, very variable in terms of histology (lymphomas, squamous cell carcinoma), but little is found about rectal adenocarcinoma concomitantly with CVID and ulcerative colitis (UC). The objective of this case report is to demonstrate the unusual association between colorectal cancer in a young patient presenting CVID.
\end{abstract}

Case presentation: A 31-years-old patient complained of an increased number of bowel movements associated with tenesmus and hematochezia and presence of mucus within the feces. As a personal history, she had been diagnosed with CVID and UC since she was 14 years old. Since then she is treated with intravenous immunoglobulin monthly and sulfasalazine, with clinical response. At the current physical examination, splenomegaly was noted and in rectal examination, a mass adhered to medium rectum was touched. The colonoscopy had revealed enanthem and ulcers, three rectal polyps and an ulcerative infiltrative lesion in the middle rectum. Biopsies identified nonspecific chronic colitis, high-grade tubular adenoma, high-grade tubulo-villous adenoma, high-grade tubular adenoma with focus of intramucosal adenocarcinoma and invasive tubular adenocarcinoma of the rectum, respectively. Staging showed no distant lesions and pelvic resonance suggested a T2N0 rectal neoplasia. The patient was then submitted to videolaparoscopic rectosigmoidectomy with total excision of the mesorectum and the pathological staging confirmed the radiological result. The patient is currently being followed by oncology, immunology, and colon and rectal surgery, asymptomatic after the re-introduction of the previous medications.

Conclusions: CVID may predispose to colorectal diseases, including IBD's and neoplasms like rectal cancer, so our article suggests these patients should be considered for clinical and endoscopic screening for the entire gastrointestinal tract disorders and not only for gastric cancers. Keywords: Rectal cancer; Common variable immunodeficiency; Ulcerative colitis; Inflammatory bowel disease

Abbreviations: CVID: Common Variable Immunodeficiency; Ig: Immunoglobulin; UC: Ulcerative Colitis; IBD: Inflammatory Bowel Disease; IUIS: International Union of Immunological Societies; ESID: European Society for Immunodeficiencies; 5-ASA: Aminosalycilates Derivatives; mm: Millimeter; cm: Centimeter; MRI: Magnetic Resonance Imaging; CT: Computerized Tomography

\section{Introduction}

There are about four hundred known monogenic inborn errors of immunity that can present various phenotypes such as susceptibility to infections, development of neoplasms, allergies, autoimmune and inflammatory diseases. The International Union of Immunological Societies (IUIS) had developed a classification based on the presentation of these diseases, in order to facilitate diagnosis and broaden consensus among publications [1].
CVID is the most common symptomatic primary immunodeficiency, predominantly related to immunoglobulin $\mathrm{G}$ and $\mathrm{A}$ deficiencies, with or without variations of IgM levels [1]. About one third of patients may also have other immune defects including $\mathrm{T}$ cells alterations [2]. The European Society for Immunodeficiencies (ESID) establishes diagnostic criteria designated in Table 1. CVID is the most heterogeneous among 
primary immunodeficiencies and may manifest in both children and adults. The B lymphocyte count may be normal, low or absent. Only $20 \%$ of CVID patients have genetic mutations identified and are no longer considered in the spectrum of this disease, but as a specific immunodeficiency, since CVID is by definition of unknown genetic cause.

Table 1: CVID diagnostic criteria by ESID.

\begin{tabular}{|c|}
\hline At least one of the following criteria: \\
\hline Increased susceptibility to infections \\
\hline Autoimmune manifestations \\
\hline Granulomatous disease \\
\hline Unexplained polyclonal lymphoproliferation \\
\hline Family member affected by antibody immunodeficiency \\
\hline $\begin{array}{c}\text { And low IgG and IgA levels with or without IgM reduction in } 2 \\
\text { measurements }\end{array}$ \\
\hline And at least one of the following: \\
\hline Poor response to vaccines (and/or absent isohemagglutinins) \\
\hline Low switched memory B-cells ( $<70 \%$ of the age-related normal value) \\
\hline $\begin{array}{c}\text { And other (primary or secondary) causes of hypogammaglobulin- } \\
\text { emia have been excluded }\end{array}$ \\
\hline And diagnosis only after four years of age \\
\hline $\begin{array}{c}\text { And absence of marked T cell deficiency defined by two of the } \\
\text { following: }\end{array}$ \\
\hline $\begin{array}{c}\text { CD4 (number/ } \mu \mathrm{L}): 2 \text { to } 6 \text { years }-<300,6 \text { to } 12 \text { years - }<250 \text { and older } \\
\text { than } 12 \text { years - }<200\end{array}$ \\
\hline $\begin{array}{c}\text { T-cell proliferation deficiency absent } \\
12 \text { years }-10 \%\end{array}$ \\
\hline
\end{tabular}

Treatment is based the immunoglobulin replacement and, in more severe cases, allogeneic bone marrow transplantation may be considered [3]. Infectious manifestations include mainly otitis, sinusitis and pneumonia, which may evolve with bronchiectasis. Infectious diarrhea by agents such as Giardia, Salmonella and Campylobacter jejuni may also occur and arthritis, notably caused by Mycoplasma, is also suggestive [3].

Another common manifestation present in up to one third of patients are autoimmune diseases, such as thrombocytopenic purpura and hemolytic anemia. In the gastrointestinal tract, celiac disease may be present as well as inflammatory bowel diseases ( 6 to $10 \%$ of the patients), with biopsy showing typical reduction of plasma cells, besides villous atrophy [4]. In addition to these diseases, CVID patients may also present Sjögren's syndrome, systemic lupus erythematosus, vitiligo, alopecia, diabetes (type 1) and Guillain-Barré syndrome.

The general incidence of cancer in CVID is about $10 \%$ and usually occurs between the fourth and sixth decade of life, with a risk between 5 to 12 times higher than in the population [2]. The most common neoplasms are B-cell lymphomas and gastric cancer. Non-Hodgkin's and Hodgkin lymphomas, pancreatic neuroendocrine tumor, chronic myeloid leukemia, breast cancer and papillary thyroid carcinoma had been described until now [3]. Lymphomas can represent up to $40 \%$ of malignancies in patients with CVID and non-Hodgkin without association with Epstein-Barr virus infection. Gastrointestinal tract tumors may occur at varying frequencies in this subpopulation $(0,48 \%$ to $14,6 \%)$, represented in $72 \%$ of the cases as gastric cancer, where Helicobacter pylori infection and pernicious anemia play important role [2].

A meta-analysis of 2016 describes colorectal involvement as $22 \%$ of gastrointestinal malignancies in CVID, but descriptions commonly do not detail the prevalent histological results. Some case reports had described intestinal involvement lymphomas, colonic neuroendocrine tumors and anal squamous cell carcinomas [5-7]. So, in this case report, we emphasize the importance of thinking about gastrointestinal involvement in CVID patients, considering that this group may have two risks factors do development of colorectal cancers: the immunological disease by itself and the IBD continuous inflammatory commitment.

\section{Case Report}

A caucasian female patient, 31-year-old, from São Paulo Brazil, complained of tenesmus, hematochezia and mucus within the stool, with a frequency of 4 evacuations daily associated with a weight loss of 13 pounds in the last 4 months. As a personal history, ate age 11, she had started do develop recurrent infections, especially sinusitis and pneumonia, as well as episodic liquid diarrhea. At 14-years-old, the diarrhea had become bloody and accompanied with a severe abdominal pain, in addition to worsening of the severity of infectious conditions, despite the emergence of arthralgia. After being diagnosed with bronchiectasis and mild pancolitis with enanthema, the patient was screened for immunodeficiencies and hypogammaglobulinemia was detected. Conduct by the immunologist, she received the diagnostic of CVID and UC. At this time, the treatment prescribed included monthly infusion of immunoglobulin and 5-ASA orally (sulfasalazine), achieving satisfactory clinical response, normalization of the bowel habits and reduction of the frequency and severity of the infectious diseases.

Over the following years, she had presented four episodes of hematemesis, diagnosed as esophageal varices and chronic liver disease. With the medical control of the portal hypertension and the endoscopic band ligation and sclerotherapy to the varicosal disease, the patient remained asymptomatic until her thirtieth birthday, having performed her last colonoscopy five years before the beginning of the symptoms with no inflammation involvement. In relation to her family history, there were no cases of polygenic, autoimmune or even neoplastic diseases.

On physical examination, the patient has adequate nutritional status, the abdomen showed a Traube space massive on percussion, besides a palpable mass in the left hypocondrium, suggesting splenomegaly. In the proctological examination, in addition to 
a small perianal tag skin, in the rectal digital examination was possible to notice a mass adhered $8 \mathrm{~cm}$ above the anal verge, occupying two third of the circumference of the middle rectum, with the presence of blood in the glove. After the emergence of the symptoms, the patient was asked to make general laboratory tests and the only altered results were a pancytopenia and a carcinoembryonic antigen of $6,7 \mathrm{mg} / \mathrm{dL}$. The colonoscopy revealed normal terminal ileum, diffuse colon enanthema, punctate ulcers with fibrin in the ascending colon, three subpediculate polyps (10 to $12 \mathrm{~mm}$ ) in upper and middle rectum (Figure $1 \& 2$ ) and an ulcerative infiltrative lesion in the middle rectum, with a diameter about $4 \mathrm{~cm}$ (Figure 3). Pathological anatomy identified nonspecific chronic colitis, high-grade tubular adenoma, highgrade tubular villus adenoma, high-grade tubular adenoma with focus of intramucosal adenocarcinoma (the specimen margins were free of neoplasia) and an invasive tubular adenocarcinoma, respectively.

Pelvic magnetic resonance imaging (MRI) showed a rectal lesion, above the peritoneal reflection, with 3,5 centimeters in diameter, occupying $60 \%$ of the circumference, invading the muscular, without compromising the mesorectal layer and without lymph node enlargement (Figure 4). Abdominal and pelvic computerized tomography (CT), in addition to the lesion described, revealed a spleen with 21 centimeters, signs of chronic liver disease and portal hypertension (Figure 5) and the chest tomography did not find any nodular lesions.
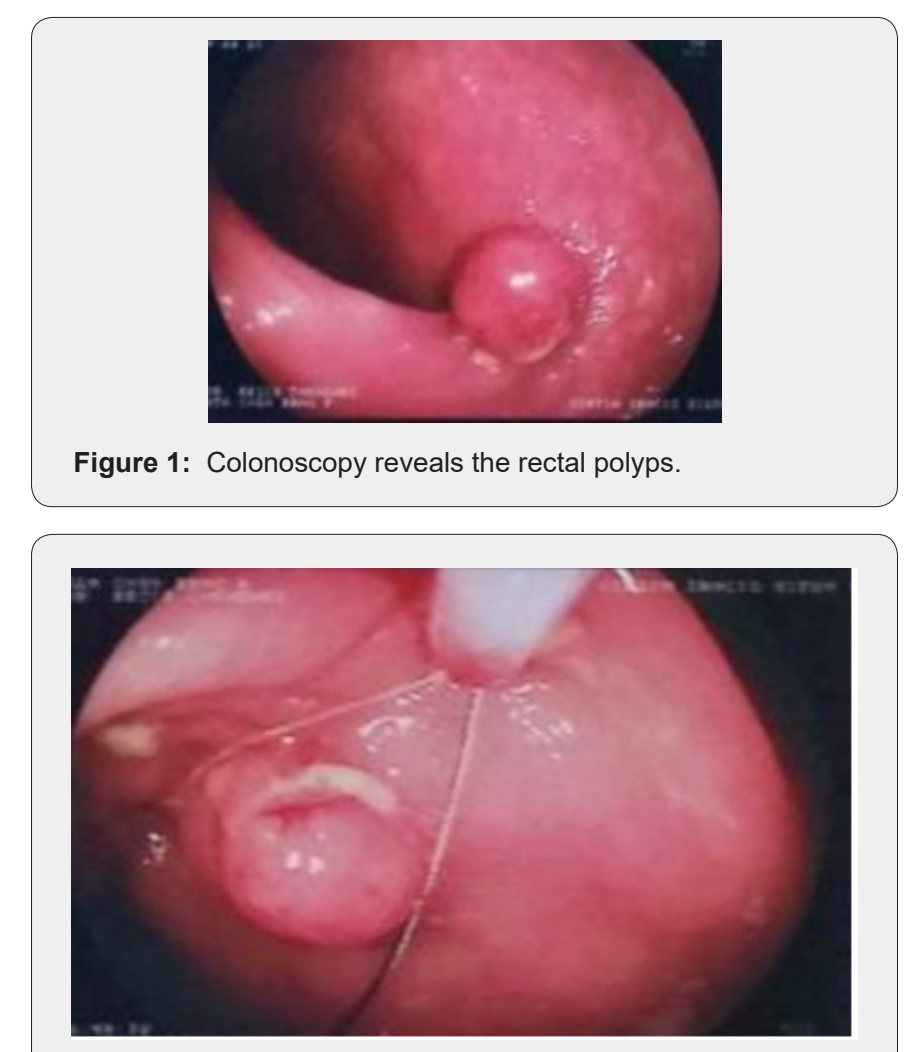

Figure 2: Colonoscopy reveals the rectal polyps.

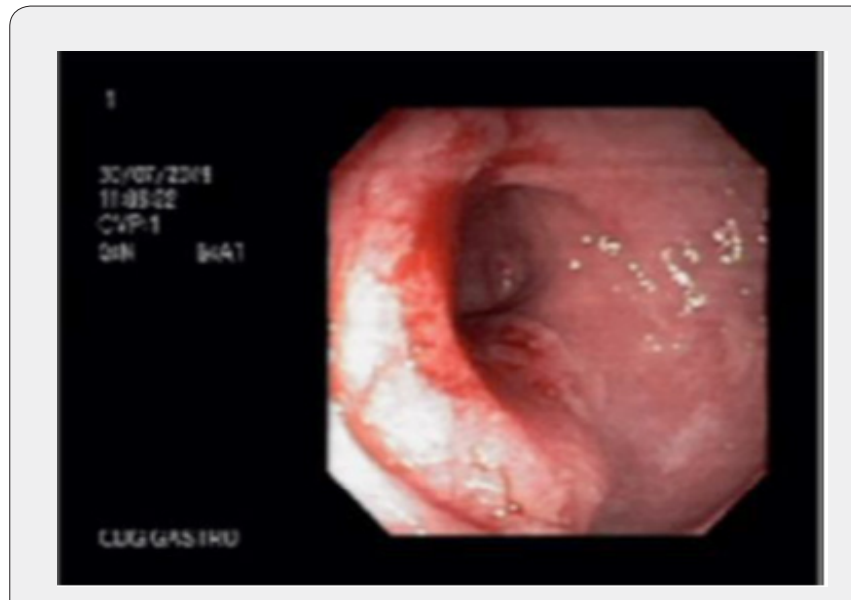

Figure 3: Colonoscopy reveals the rectal tumor.

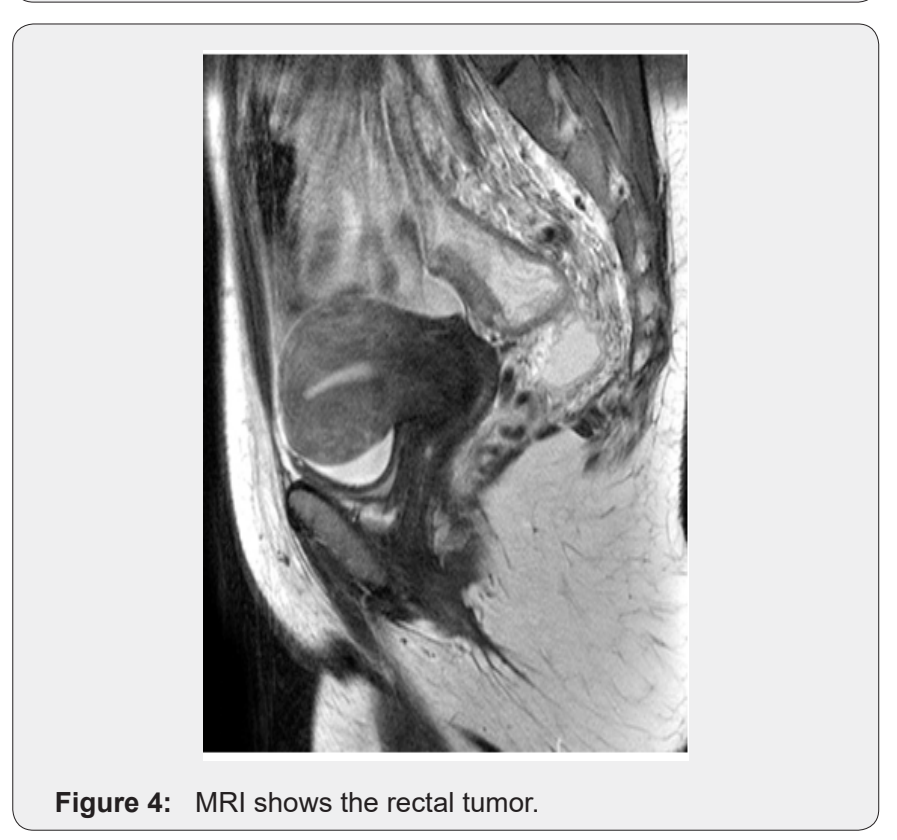

Figure 4: MRI shows the rectal tumor.

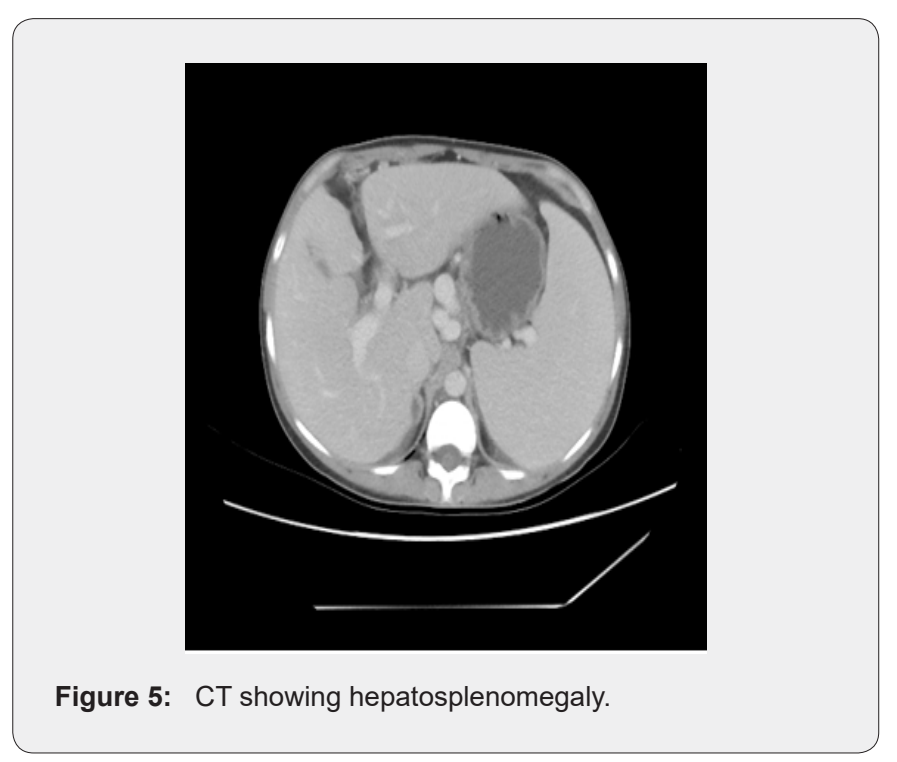


The patient underwent laparoscopic abdominal rectosigmoidectomy with total mesorectal excision, as the initial stage was T2N0M0 and the lesion coincident with the limit of peritoneal reflection (Figure 6\&7). The histological conclusion was a moderately invasive carcinoma of 2,6 cm, pT2pN0, with thirteen lymph nodes unaffected. The postoperative course continued without any complications and, despite the mayor susceptibility, there were no infectious diseases, with diet reintroduction on the first postoperative day. Currently, the patient has been followed by a multidisciplinary team (immunologist, oncologist and colon and rectal surgeon) for eighteen months without signs of cancer relapse. 5- ASA derivative was prescribed again, as well as immunoglobulin and the beta-blocker to the portal hypertension and until now the patient remains asymptomatic.

\section{Discussion}

This case report illustrates the rare occurrence of rectal adenocarcinoma in a patient with CVID. It is well known that these patients most commonly develop autoimmune diseases such as UC and this condition may predispose to colorectal cancer after some years of chronic inflammation, however there are no publications about this evolution in patients with a primary immunodeficiency. This case may suggest that in some groups like this, gastrointestinal cancer screening should not only be done to the upper tract, with upper endoscopy, due to the mayor incidence of gastric cancer. Careful attention should be paid to complaints related not only to colorectal cancer, but also to IBD, proposing an early diagnose and assertive treatment, with a follow-up with smaller intervals between the endoscopic examinations, since the malignancies seems to develop early in these patients. Some reports have referred to intestinal involvement lymphomas, colonic neuroendocrine tumors and anal squamous cell carcinoma [5-7], but until this article writing nothing was found about rectal adenocarcinoma in the indexed publications.

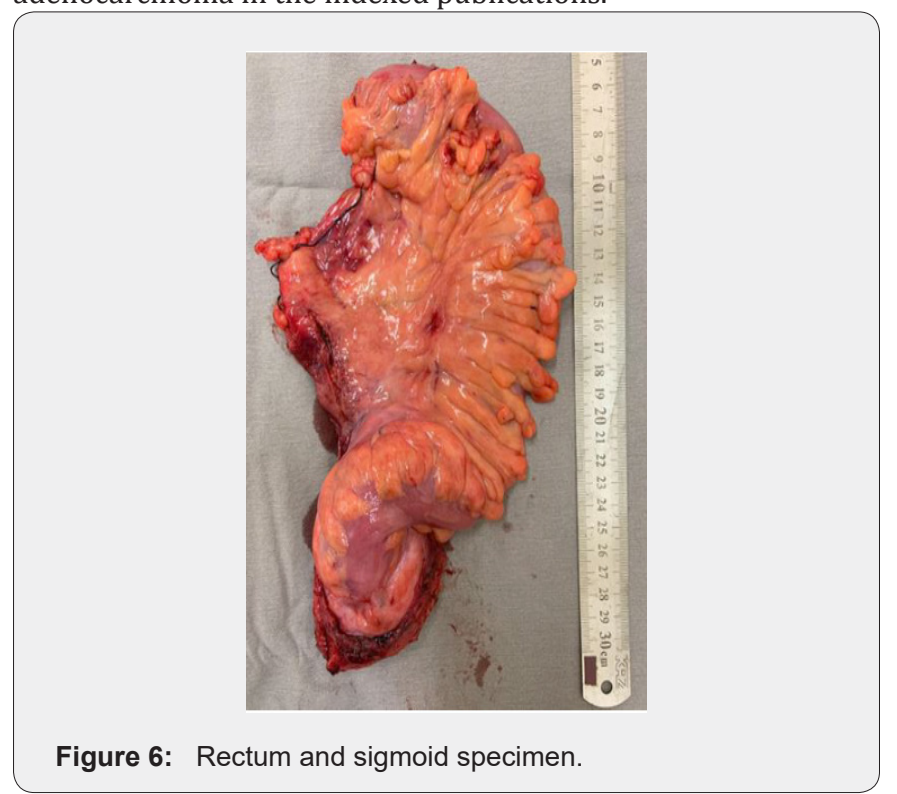

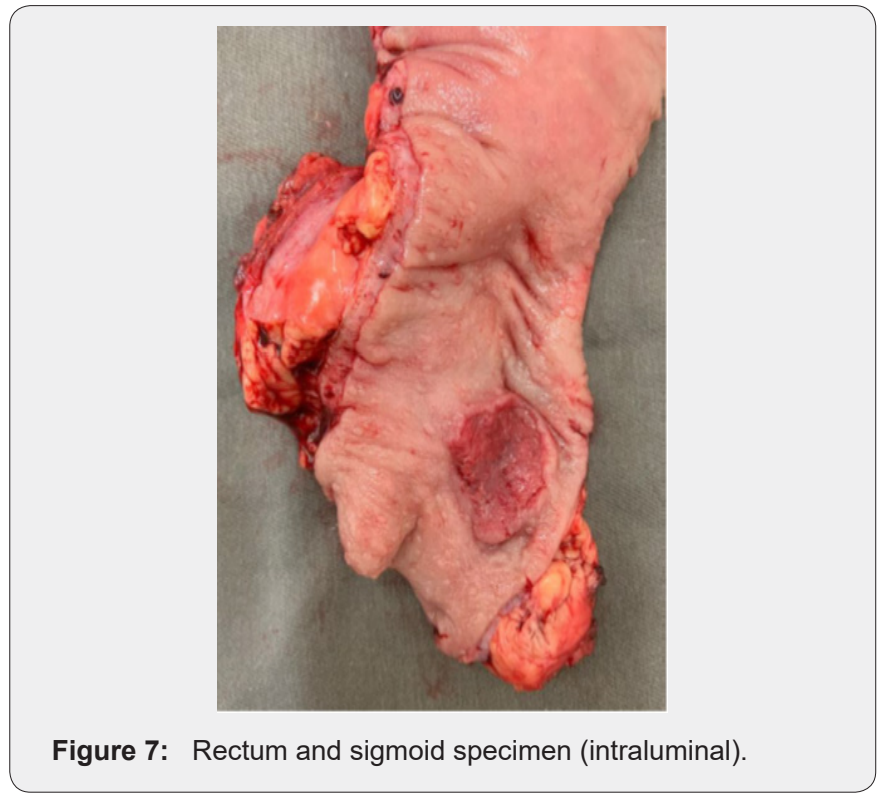

\section{Conclusion}

In conclusion, we suggest that patients with CVID should undergone early endoscopic screening of the upper and low gastrointestinal tract, aiming to diagnose IBD or other predisposing malignant conditions.

\section{Declarations}

\section{Ethics approval and consent to participate}

This case report was submitted to the ethical committee "Comitê de Ética para Análise de Projetos de Pesquisa" (CAPPESQ) of the Hospital das Clínicas - Faculdade de Medicina da Universidade de São Paulo and to Plataforma Brasil, with approval. The informed consent signed by the patient is available for consultation.

\section{Competing Interests and Funding}

The authors declare that this article does not have any competing interest and did not receive any funding specifically intended to accomplish this article.

\section{Availability of Data and Materials}

Data sharing is not applicable to this article as no datasets were generated or analysed during the current study, because it is a case report in which all relevant exams and patient's information is available in the article itself.

\section{Author's Contribution}

Vivian Regina Guzela assisted Carlos Sobrado in the treatment of this patient, gathered and reviewed the clinical data of the case report and wrote this article. 
Carlos Walter Sobrado Junior wrote this article and was responsible for the treatment of the patient, especially for the surgical approach.

Dewton de Moraes Vasconcelos reviewed this article and was responsible for the treatment of the patient, mainly the immunological approach.

Lucas Faraco Sobrado assisted Carlos Sobrado in the treatment of this patient and was responsible for literature review.

Sérgio Carlos Nahas reviewed this article and was responsible for providing part of the institutional resources as a chief of the Colon and Rectal Surgery Division.

Ivan Cecconello reviewed this article and was responsible for providing part of the institutional resources as a chief of the Gastroenterology Department.

\section{References}

1. Bousfiha A, Jeddane L, Picard C, Ailal F, Bobby Gaspar H, et al. (2018) The 2017 IUIS Phenotypic Classification dor Primary Immunodeficiencies. J Clin Immunol 38(1): 129-143.

2. Kiaee F, Azizi G, Rafiemanesh H, Zainaldain H, Sadaat Rizvi F, et al. (2019) Malignancy in common variable immunodeficiency: a systematic review and meta-analysis. Expert Rev Clin Immunol 15(10): 1105-1113.

3. Saikia B, Gupta S (2016) Common variable immunodeficiency. Indian J Pediatr 83(4): 338-344.

4. Kelsen JR, Sullivan KE (2017) Inflammatory bowel disease in primary immunodeficiencies. Curr Allergy Asthma Rep 17(8): 57.

5. Montálban C, Calleia JL, Bellas C, Erice A, Patier JL, et al. (1989) Lymphoma of the rectum associated with lymphoid nodular hyperplasia in a common variable immunodeficiency. Med Clin (Barc) 93(10): 377-379.

6. Gemeinhardt M, Türck J, Piper B, Helmberger T, Nerlich A, et al. (2012) Adenocarcinoma of the stomach and neuroendocrine carcinoma of the colon in a 45-year old male patient suffering from common variable immunodeficiency (CVID) and ulcerative colitis. Z Gastroenterol 50(12): 1292-1295.

7. Goldberg A, Lewis R, Mccue P (2016) Common variableimmunodefiency disease and rectal squamous cell carcinoma: a case report of a rare syndromic tumor type. Gastrointest Cancer 47(4): 470-473.

\section{Your next submission with JuniperPublishers will reach you the below assets}

- Quality Editorial service

- Swift Peer Review

- Reprints availability

- E-prints Service

- Manuscript Podcast for convenient understanding

- Global attainment for your research

- Manuscript accessibility in different formats

( Pdf, E-pub, Full Text, audio)

- Unceasing customer service

Track the below URL for one-step submission https://juniperpublishers.com/online-submission.php 